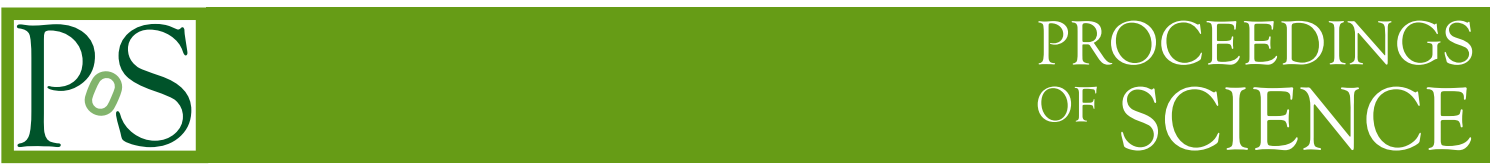

\title{
Performance Evaluation of Geant4 Particle Therapy System Simulation Framework in Grid Environments
}

\footnotetext{
Tsukasa Aso, ${ }^{a b}$ Wataru Takase, ${ }^{b}$ Go Iwai, ${ }^{b}$ Takashi Sasaki, ${ }^{b}$ Yoshiyuki Watase, ${ }^{b}$ Yoshikazu Maeda, ${ }^{c}$ Tomohiro Yamashita, ${ }^{d}$ Takashi Akagi, ${ }^{d}$ Harada Shuuichi, ${ }^{e}$ Teiji Nishio, ${ }^{f}$ Siou-Yin Cai, ${ }^{g}$ Tsi-Chian Chao, ${ }^{g}$ Eric Yen, ${ }^{h}$ Tsung-Hsun Wu, ${ }^{h}$ Yu-Tsun Lin, ${ }^{h}$

${ }^{a}$ Nagaoka University of Technology

1603-1 Kamiotmioka-machi, Nagoka, Niigata 9402188, Japan

${ }^{b}$ High Energy Accelerator Research Organization (KEK)

1-1 Oho, Tsukuba, Ibaraki 3050801, Japan

${ }^{c}$ Fukui Prefectural Hospital Proton Therapy Center

2-8-1 Yotsui, Fukui, Fukui 9108526, Japan

${ }^{d}$ Hyogo Ion Beam Medical Center

1-2-1 Koto Shingu-cho, Tatsuno-shi, Hyogo 6795165, Japan

${ }^{e}$ Hyogo Ion Beam Medical Support

1-2-1 Koto Shingu-cho, Tatsuno-shi, Hyogo, 6795165, Japan

${ }^{f}$ National Cancer Center Hospital East

6-5-1 Kashiwanoha, Kashiwa, Chiba 2778588, Japan

${ }^{g}$ Chang Gung University

259 Wen-Hwa 1st Road, Kwei-Shan, Tao-Yuan 333, Taiwan

${ }^{h}$ Academia Sinica Grid Computing Centre (ASGC)

2128 Academia Road, Section 2, Nankang, Taipei 11529, Taiwan

E-mail: aso@nagaokaut.ac.jp, wataru.takase@kek.jp, go.iwai@kek.jp,

Takashi.Sasaki@kek.jp, yoshiyuki.watase@kek.jp,

y-maeda-ce@pref.fukui.lg.jp, t.yamashita@ham-progress.jp,

t.akagi@ham-progress.jp, s.harada@ham-progress.jp,

tnishiodeast.ncc.go.jp, sycai@mail.cgu.edu.tw,

chaotemail.cgu.edu.tw, Eric.Yen@twgrid.org, tsung-hsunetwgrid.org,

YT.Linetwgrid.org ${ }^{\dagger}$
} 
The PTSIM (Particle Therapy System Simulation Framework) is a Monte Carlo simulation framework based on Geant 4 for particle therapy validated with protons and carbons. Although the Monte Carlo simulation is believed to be the most reliable method of dose calculation, the calculation time is still critical in performing the simulation with suff cient statistical accuracy for clinical applications. The grid computing environments provide a straightforward way to gain computational speed without modifying the current PTSIM code. We have studied the performance of PTSIM in grid computing environments. The system parameters such as turnaround time, waiting time, simulation time and data transfer time have been collected in each simulation for different treatment conf gurations and grid computing environments. The simulation time was examined in conditions for the validation of treatment port and the quality assurance of clinical applications, respectively. In order to reduce the simulation time for clinical applications, we prepared the phase space data of tracks at the entrance of treatment head in validated treatment port and repeated the simulation by generating particles in the phase space data. In this paper, we report on the result of scalability benchmark for these simulations in treatment port validations and quality assurance in clinical applications.

International Symposium on Grids and Clouds (ISGC) 2014,

23-28 March 2014

Academia Sinica, Taipei, Taiwan

\footnotetext{
*Speaker.

${ }^{\dagger}$ Authors would like to thanks Professor Augastine Ei-Foung Chen of National Central University and the members of TWGRID, especially Zong-Tsung Wu and Jhen-Wei Huang for the valuable support, suggestions and comments.

c Copyright owned by the author(s) under the terms of the Creative Commons Attribution-NonCommercial-ShareAlike Licence.
} 


\section{Introduction}

The particle therapy is one of external beam radiotherapies for cancer treatment, which uses hadron beam of protons or heavy-ions. The mono-energetic hadron beam stops at a certain depth in a substance and deposits a large portion of its energy near the end of its range. This physical characteristic is known as the Bragg peak and suitable for radiotherapy, because it delivers localized dose to tumor while minimizing damage to healthy tissues. Since the dose distribution inside patient can not be measured directly, dose calculation is essential to ensure patient's safety and effect of treatment. Therefore, there is a need to develop reliable simulation tools of dose calculation corresponding to the progress in radiotherapy.

The Geant $4[1,2]$ is a software toolkit that is used to simulate the interaction of particles in matter and has been widely used in various field from high-energy physics to nuclear physics to space and medicine. Since Monte Carlo simulation using Geant4 simulates the interaction of particles in matter by taking into account the physics based on theoretical models or experimental cross-section data, it is considered to be the most accurate method to calculate the dose in radiotherapy.

The particle therapy simulation framework (PTSIM) $[3,4,5,6]$ is a Geant4 based Monte Carlo simulation framework for particle therapy validated with protons and carbon-ions. The PTSIM provides a common platform to model treatment port including a patient geometry from CT images, allowing users who are not Geant 4 experts to accurately and efficiently run Geant 4 simulations. The PTSIM can be helpful in improvement of treatment plans and quality assurance of ongoing treatments.

Although the PTSIM has been heavily used in medical physics research, clinical applications remain limited since the simulation time is critical in performing dose calculation with sufficient statistical accuracy. In order to gain computation speed, the parallelization of simulation in grid computing environments is a straightforward way without modifying the current PTSIM code. In this paper, we report on the study of scalability of PTSIM on grid environments.

\section{Overview of the PTSIM}

The PTSIM was developed in the project, "Development of simulation framework for advance radiotherapy", funded by the Japan Science and Technology Agency (JST) in the program of Core Research for Evolutional Research and Technology (CREST), 2003 to 2010. Efforts on further development of PTSIM are still underway to include more functionality and improve the performance.

The PTSIM is a single software application for modeling a treatment port consisting of a beam delivery system and a treatment head with a patient data obtained from CT images. The configuration of treatment port is defined by a series of parameters for beam devices, a primary beam, physics processes and a target object. Online commands are provided for modifying the parameters to configure a customized treatment port. All beam devices are referred to as beam modules in the PTSIM and only beam modules selected by users are placed in the beam line and configure a treatment port. Therefore, users are not requested to develop program code. The PTSIM can import CT images in Digital Imaging and Communications in Medicine (DICOM) format [7] and can 


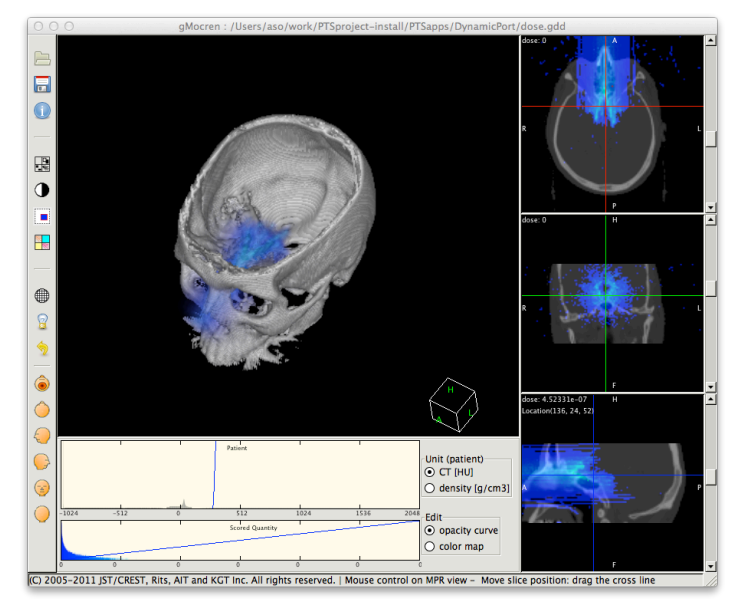

Figure 1: An example of dose distribution in a sample patient geometry from CT images.

construct a patient geometry by placing voxels filled with materials corresponding to Hounsfield units (HU) in CT images. Fig.1 shows an example of dose distribution for sample CT images. The DICOM format for treatment parameters are defined as one of sub-categories in the extension of the DICOM format for radiotherapy (DICOM-RT). The RT-IonPlan describes treatment parameters for ion therapy and includes parameters of the treatment port and the patient registration. However, the meanings of some parameters in RT-IonPlan are specialized to a specific institution. Therefore the PTSIM currently accepts treatment parameters in DICOM-RT format in Hyogo Ion Beam Medical Center (HIBMC), Fukui Prefectural Hospital Proton Therapy Center (FPHPTC), Gunma University Heavy Ion Medical Center (GHMC) and Nagoya Proton Therapy Center (NPTC). Fig.2 shows the example of treatment port and the treatment head geometries according to the treatment parameters in sample RT-IonPlan data in FPHPTC.

The dose distributions in PTSIM was validated with measurements for particle therapy using protons and carbon-ions $[8,9]$. In addition to the validations of treatment ports, PTSIM is used for quality assurance of ongoing treatment such as verification of biological dose calculation for cabon ion therapy [10], study on the effect of inhomogeneity in a patient's body [11] etc.

\section{Materials and Methods}

\subsection{Overview of grid environments}

We used computing grid environments in KEK central computing system (KEKCC) and Taiwan grid computing system (TWGrid). The KEKCC is a shared computing environment among users for high energy physics experiments etc. The computing environment we used in TWGrid was experimentally configured as a test bench system for this study. Because of the total number of cores in the test bench system in TWGrid was limited up to 200, we utilized it for checking our developed job submitting system that a user can submit a job on the common user interface from KEKCC to either KEKCC or TWGrid environments. The scalability test has been performed in the KEKCC environment. 

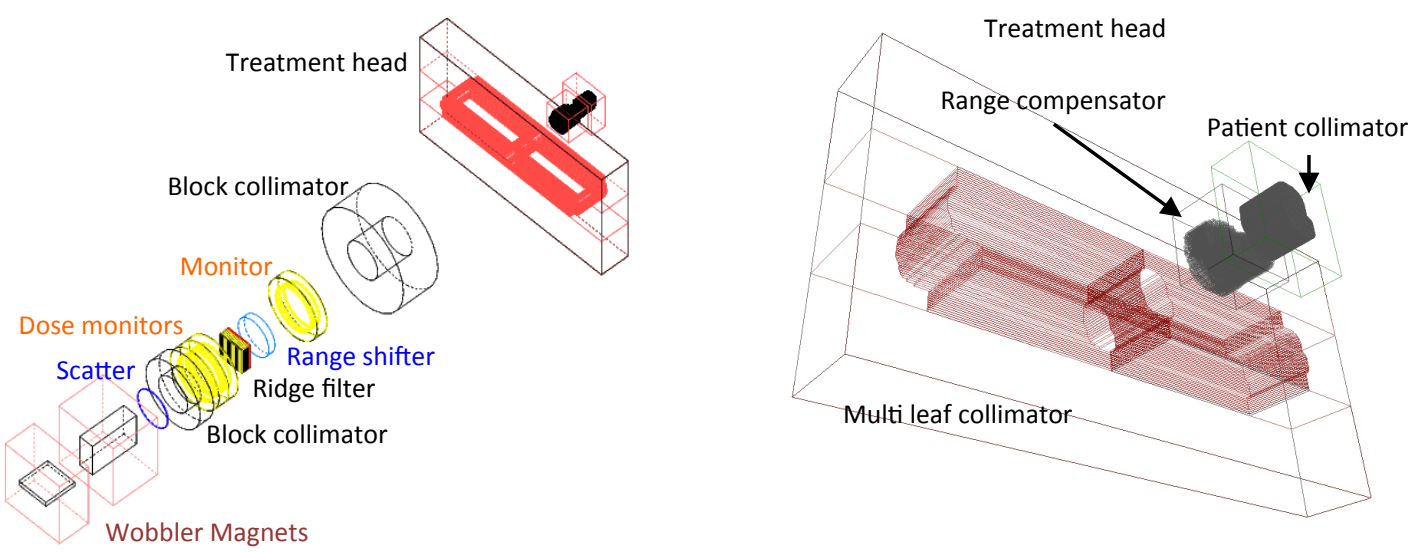

Figure 2: The example geometries of treatment port (left) and treatment head (right) in PTSIM. The beam devices are configured corresponding to the treatment parameters in RT-IonPlan data.

Fig. 3 shows a schematic diagram of computing environments. The Geant4 libraries, a PTSIM executable and CT images had been preinstalled in shared disk space of each environment, while our job submitting system was installed only in KEKCC. The job submitting system works for (1) transferring data files and macro files needed for the PTSIM simulation from user's home directory in KEKCC to the shared disk space in the computing environment, (2) submitting PTSIM tasks to the batch job management system in the computing environment, (3) starting a monitoring process and watching the status of PTSIM tasks in the batch job management system, (4) starting a merging process after completing all PTSIM tasks to integrate the results of PTSIM tasks into a three dimensional dose distribution, and finally (5) transferring the integrated dose distribution to the user's home directory in KEKCC. Here the batch job management systems used in KEKCC and TWGrid are the load sharing facility (LSF) and the portable batch system (PBS), respectively. The job submitting system records elapsed time stamp at each step so that the time period of each process can be derived. Fig. 4 demonstrates time charts of example jobs for calculating the dose distributions in a water phantom in a proton treatment port.

\subsection{Simulation conditions}

We performed simulations for the proton treatment port in FPHPTC. The treatment port comprises a wobbler magnet and a scatter as a lateral beam system, a ridge filter as a range modulator to make spread-out Bragg peak, a range shifter as a fine degrader and a multi-leaf collimator (MLC). The patient-specific range compensator and collimator are optionally equipped depending to the treatment plan. There are monitors and block collimators on the beam line in addition to those devices.

We prepared artificial treatment plan data files for head-neck in small and large fields, liver and prostrate cancers by using the treatment planning system in hospital information system. We adopted a water phantom from CT images as the target geometry by reforming $2 \mathrm{~mm}$ cubic voxels. Table 1 shows representative parameters in the treatment plans. The treatment plans in RTIonPlan format depends on the facility specifications, while we deployed the PTSIM to the computing 


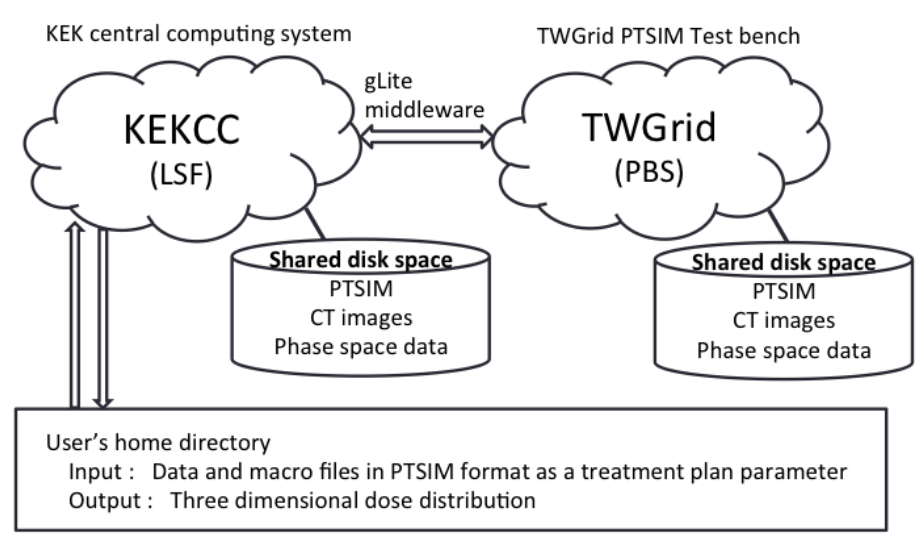

Figure 3: The schematic diagram of computing environments.

\begin{tabular}{l|c|c|c|c}
\hline Types & $\begin{array}{c}\text { Head-neck } \\
\text { Small field }\end{array}$ & $\begin{array}{c}\text { Head-neck } \\
\text { Large field }\end{array}$ & Liver & Prostate \\
\hline \hline Beam Energy (MeV) & 150 & 190 & 190 & 235 \\
SOBP $(\mathrm{cm})$ & 3 & 10 & 8 & 8 \\
Range shifter (cm) & 4.9 & 5.4 & 3.2 & 8.3 \\
Bolus & used & used & used & used \\
Patient collimator & used & N/A & used & N/A \\
\hline
\end{tabular}

Table 1: The signature of configuration parameters applied for PTSIM simulations.

environments as a common simulation framework independent to specific facilities. Therefore, we translated the treatment plan data in RTIonPlan format into PTSIM format and saved it in parameter data files and command files for PTSIM simulations.

The performance was examined in conditions for the validation of treatment port and the quality assurance of clinical applications. The protons were simulated from the beam extract window to the water phantom at the isocenter for validating the treatment port. In order to reduce the simulation time for clinical applications, we recorded track information in front of MLC in the validated treatment port and reused it to repeat simulations in the patient specific area consisting of the treatment head and the patient geometry. The track information of photons, electrons, positrons, neutrons and protons were written in the IAEA phase space data format. By starting simulations in front of patient specific area, we can consolidate redundant simulations of the beam delivery system.

\section{Results}

As shown in Fig.4, the turnaround time varies with the waiting time in the queue of batch job management system, and it depends on the policy of job management system such as the availabil- 


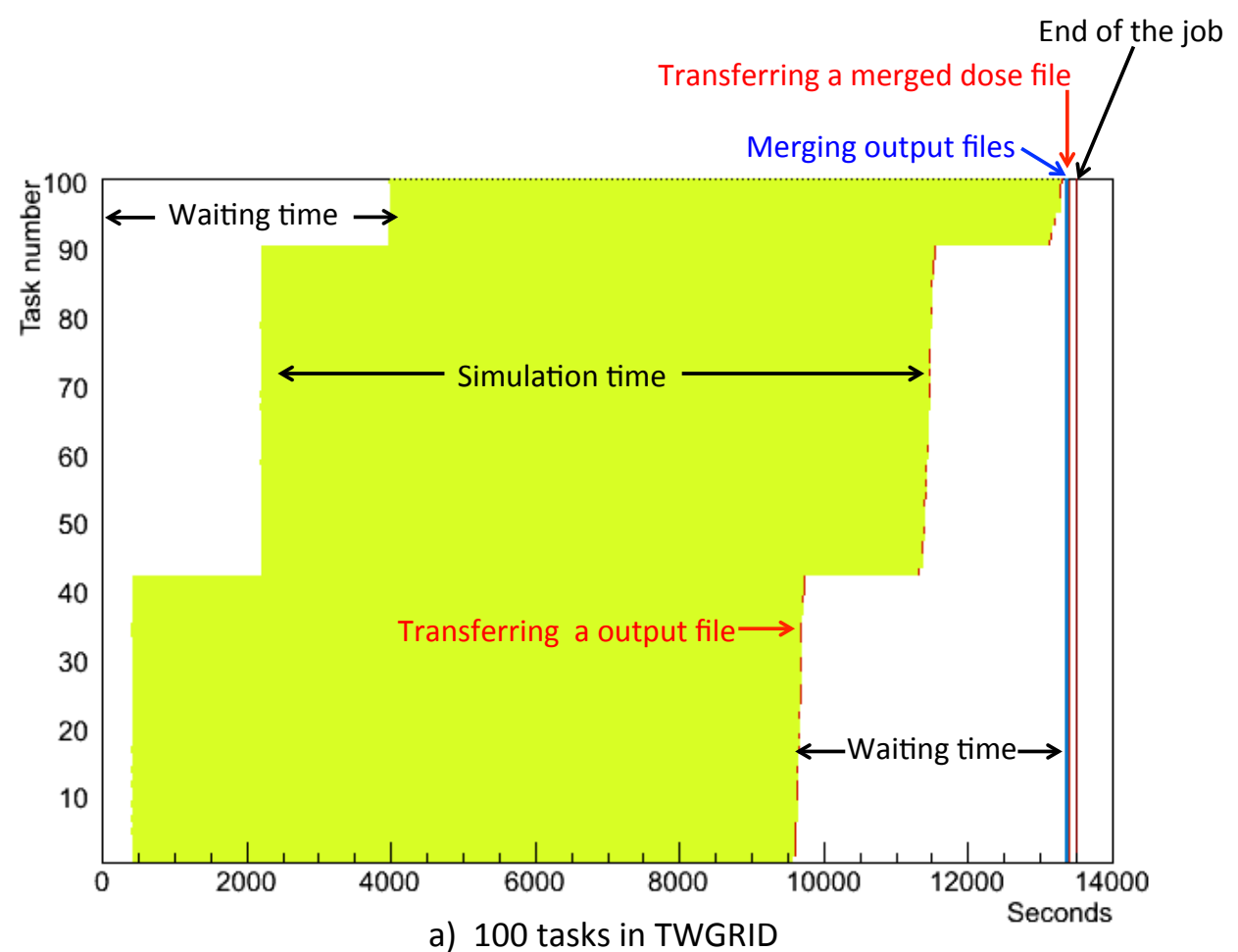

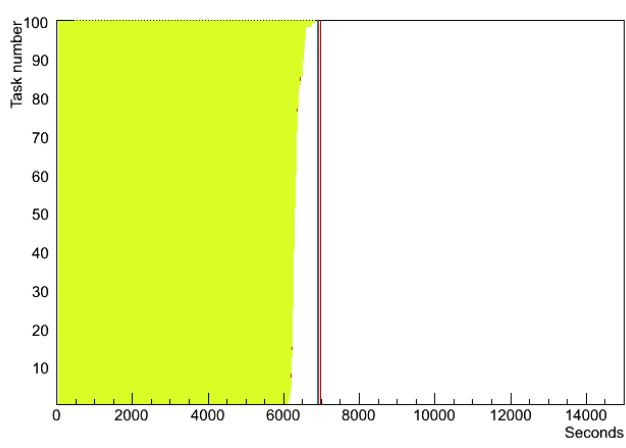

b) 100 tasks in KEKCC

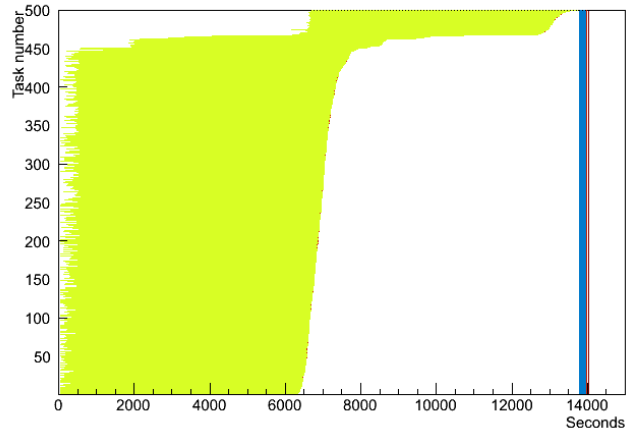

c) 500 tasks in KEKCC

Figure 4: Example time charts for calculating a dose distribution in a water phantom in a proton treatment port. (a) a job consisting of 100 PTSIM tasks was submitted to the TWGrid environment, (b) a job consisting of 100 PTSIM tasks was submitted to the KEKCC environment, and (c) a job consisting of 500 PTSIM tasks was submitted to the KEKCC environment. Each task simulated $1.8 \times 10^{6}$ primary protons. The horizontal and vertical axes show the time elapsed from the job submission $(\mathrm{t}=0 \mathrm{~s})$ and the sequential task number, respectively. As indicating in (a), the white blank area represents inactive state such as that the task in waiting queue. The green area shows the actual simulation time periods of PTSIM tasks. The red area attaching to the end of green area represents the time period for transferring the output data file from the local disk in computing node to the shared disk space. The blue area represents the time period for merging output data files to a dose data file. The red area attaching to the end of blue area represents the time period for transferring the merged dose data file to user's home directory in KEKCC. Finally, the last red line, for example the line around $13000 \mathrm{~s}$ in (a), represents the time of end of the job. 


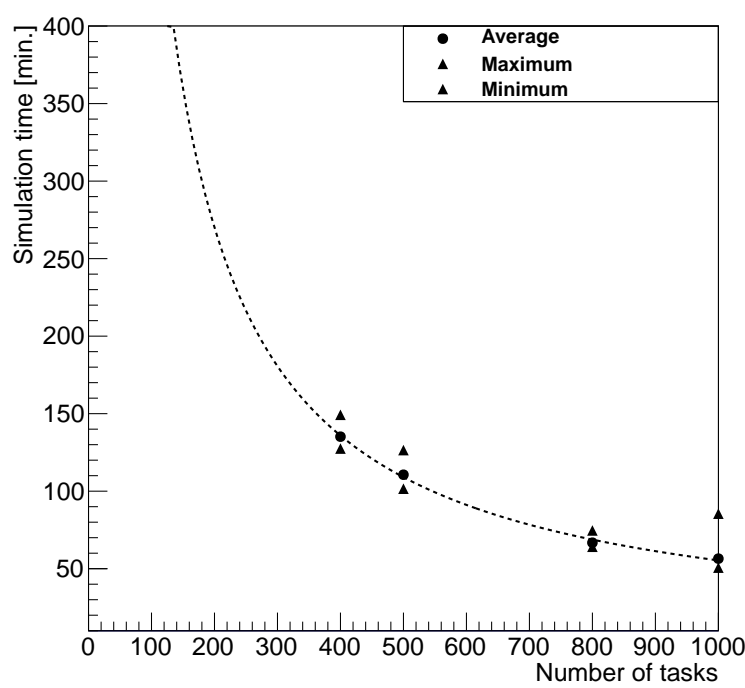

Figure 5: Simulation time of PTSIM tasks as a function of number of tasks for simulating $9 \times 10^{8}$ primary protons. Therefore, each PTSIM task in 400, 500, 800, and 1000 tasks simulates $2.5 \times 10^{6}, 1.8 \times 10^{6}$, $1.125 \times 10^{6}$, and $0.9 \times 10^{6}$ primary protons, respectively. The simulation time is given as the average of all PTSIM tasks, the minimum and the maximum simulation time in the PTSIM tasks.

ity of computing resource and the priority of the task. Therefore, we focused on the simulation time of PTSIM in following discussions.

The CPU performances of computing resources were evaluated by submitting jobs by varying the number of primary protons per PTSIM task. Here each job contained 10 PTSIM tasks. The average simulation times per primary proton were about $3.3 \mathrm{~ms}$ and $5.1 \mathrm{~ms}$ in KEKCC and TWGrid environments, respectively.

\subsection{Scalability}

We studied the scalability of simulation time in the KEKCC environment by varying the number of PTSIM tasks to 400,500, 800 and 1000, while keeping the total number of primary protons to $9 \times 10^{8}$. Therefore, each PTSIM task simulates $2.5 \times 10^{6}, 1.8 \times 10^{6}, 1.125 \times 10^{6}$, and $0.9 \times 10^{6}$ primary protons, respectively. The total number of primary protons was chosen to achieve sufficient statistical accuracy within $2 \%$ in three-dimensional dose distribution. The primary protons were simulated from beam exit window to the target at the isocenter. The treatment port was configured according to the sample treatment plan data for the head-neck with small field cancer. Fig.5 shows the scalability of average simulation time of PTSIM tasks. The dashed line in the figure was obtained by fitting data points with $T=T_{0}+T_{1} / N_{\text {Task }}$, where $T$ and $N_{\text {Tasks }}$ are the average simulation time and the number of tasks, and $T_{0}$ and $T_{1}$ are fitting parameters, respectively. The $T_{0}$ and $T_{1}$ were obtained as $(100 \pm 183) \mathrm{s}$ and $(3.2 \pm 0.1) \times 10^{6} \mathrm{~s}$, respectively. The reduction of simulation time scales with the number of tasks. The simulation time in one CPU was estimated about 890 CPU hours. 


\begin{tabular}{ll|ccc}
\hline $\begin{array}{l}\text { Treatment } \\
\text { configuration }\end{array}$ & $\begin{array}{c}\text { Full treatment port } \\
\text { [min.] }\end{array}$ & $\begin{array}{c}\text { Treatment Head } \\
\text { [min.] }\end{array}$ & $\begin{array}{c}\text { Ratio } \\
{[\%]}\end{array}$ \\
\hline Head-neck & small field & 108 & 33 & 31 \\
Head-neck & large field & 117 & 47 & 40 \\
Liver & & 205 & 141 & 67 \\
Prostate & & 119 & 49 & 41 \\
\hline
\end{tabular}

Table 2: Average run time of PTSIM in treatment configuration for the head-neck in small field and large field,

\subsection{Treatment port configurations and IAEA phase space data}

Table 2 shows the average simulation time of PTSIM tasks in the treatment plans. Here the number of primary protons and number of PTSIM tasks per job was fixed to $9 \times 10^{8}$ and 500 , respectively. The average simulation times were studied for the simulations in the full treatment port volume and the treatment head volume by using phase space data. The former and latter conditions correspond to the simulations for the validation of treatment port and the quality assurance of clinical applications, respectively. By using IAEA phase space data, the average simulation time was effectively reduced.

\section{Summary}

We have studied the scalability of PTSIM in grid environments. The job submitting system was developed that user can submit a job from KEKCC to either KEKCC or TWGrid environments with the common user interface. The scalability of PTSIM in the number of PTSIM tasks was confirmed up to 1000 PTSIM tasks. In addition, we confirmed that separating the simulation using phase space data effectively reduced the average simulation times.

\section{References}

[1] S.Agostinelli et al., Geant4, a simulation toolkit, Nucl., Instr. Meth. Res., A506, 250-303 (2003).

[2] J. Allison et al., Geant4 Developments and Applications, IEEE Trans. Nucl. Sci., 53, no. 1, 270-278 (2006).

[3] T.Akagi, T.Aso et al., The PTSim and TOPAS Projects, Bring- ing Geant4 to the Particle Therapy Clinic, Prog. in Nucl. Sci. Technol., 912-917 (2011).

[4] T.Akagi, T.Aso, G.Iwai et al., Geant4-based particle therapy simulation framework for verification of dose distributions in proton therapy facilities, Progress in Nuclear Science and Technology, Accepted for publication.

[5] T.Aso, Y.Maeda, G.Iwai et al., Extension of the particle therapy simulation framework to hospital information systems and multi-grid environments, IEEE International Conference on Computing and Engineering, IEEE Computer Society, 229-234 (2012)

[6] G. Iwai, W.Takase, T.Aso et al., Common platform of Monte Carlo dose calculation on universal grid interface with Geant4 based particle therapy simulation framework, XVII International Conference on 
the Use of Computers in Radiation Therapy (ICCR 2013) IOP Publishing, Journal of Physics: Conference Series 489 (2014) 012001.

[7] DICOM, Digital Imaging and Communications in Medicine, from the National Electrical Manufactures Association [On- line], Available: http://medical.nema.org/.

[8] T. Aso et al., Verification of the dose distributions with GEANT4 simulation for proton therapy, IEEE Trans., Vol. 52, Issue 4, 896-902 (2005).

[9] S.Kameoka et al.,Dosimetric evaluation of nuclear interaction models in the Geant4 Monte Carlo simulation toolkit for carbon-ion radiotherapy, Radiol. Phys. Tehnol., 1, 183-187 (2008).

[10] H.Nose, T.Aso, Y.Kase, N.Matsufuji, T.Kanai, Verification of biological dose calculation for carbon ion therapy with a Monte Carlo method, Japanese Journal of Medical Physics, Vol. 28 no. 4, 132-141 (2009).

[11] T.Yamashita, T.Akagi, T.Aso, A.Kimura, T.Sasaki, Effect of inhomogeneity in patientś body on the accuracy of pencil beam algorithm in comparison to Monte Carlo, Phys. Med. Biol. 57 7673-7688 (2012).

[12] IAEA INDC (International Nuclear Data Committee) phase-space database for external beam radiotherapy, 2005 INDC(NDS) 04841. 